International Journal of Library \& Information Science (IJLIS)

Volume 7, Issue 1, January-February 2018, pp. 82-93, Article ID: IJLIS_07_01_011

Available online at

http://iaeme.com/Home/issue/IJLIS?Volume=7\&Issue=1

Journal Impact Factor (2016): 8.2651 (Calculated by GISI) www.jifactor.com

ISSN Print: 2277-3533 and ISSN Online: 2277-3584

\title{
AWARENESS AND USE OF OPEN ACCESS RESOURCES AMONG THE STUDENTS OF SELECTED ENGINEERING INSTITUTIONS IN TELANGANA STATE: A SURVEY
}

\author{
Jagadish, B \\ Research Scholar, Department of Library and Information Science, \\ Dravidian University, Kuppam, Andhra Pradesh \\ Anjaiah, M \\ Research Supervisor, Department of Library and Information Science, \\ Dravidian University, Kuppam, Andhra Pradesh
}

\begin{abstract}
The study is an attempt of assessing the awareness and use of open access resources among the students of selected engineering institutions in Telangana state by a survey of 766 participants through a questionnaire. It was found that the level of awareness on the open access resources meant for academic related activities is quite encouraging. In addition, the open access resources accessible through online have been well utilized. The use of academic social networking sites, open access journals, e-books, online lecture videos, etc was found to be quite encouraging. However, a meager portion of the respondents expressed that they did not use open access resources while another portion of the respondents revealed that they were not even aware of some open access resources. Majority of the respondents have used very little some important sources which are very essential for academic and research purposes such as Plagiarism Detection Tools, Manuscript Editing Tools, and Reference Management Tools. The respondents also had some constraints in using open access resources such as inadequate search facility, Lack of up-to-date information, Slow and hidden feedback, Lack of subject coverage, Nonavailability of full text, and Lack of Speed.
\end{abstract}

Keywords: open access resources, social media, Telangana State, engineering institutions, electronic information resources, digital information sources

Cite this Article: Jagadish, B and Anjaiah, M, Awareness and Use of Open Access Resources among the Students of Selected Engineering Institutions in Telangana State: A Survey. International Journal of Library \& Information Science, 7(1), 2018, pp. 82-93. http://iaeme.com/Home/issue/IJLIS?Volume=7\&Issue=1 


\section{INTRODUCTION}

It is a well-known fact that there was a huge difference in the information access between information haves and information have-not's. It happened due to the non-availability of information particularly the scholarly information for the people who were really with innovative ideas with an intention to transform the society through new innovations. Due to non-availability of sufficient information in their field of interest or unaffordable situation of an individual or the organization concerned with the commercial publishers, the researchers were not in a position to implement/execute their innovative ideas. But the situation today is entirely different due to the advent of Information and Communication Technology (ICT) which has influenced all walks of life. Emergence of ICT has reshaped the publication industry to make the scholarly communication available in many ways. On the other hand, growth of scientific literature exponentially has posed a set of challenges to the information providers in providing access to vast amount of scientific information. Moreover, unrealistic increase in the prices of academic journals by the publishers was an added challenge to the libraries and researchers to conduct fruitful research in the emerging areas.

\section{PROBLEM STATEMENT}

It is well known fact that the advent of Information and Communication Technology (ICT) has influenced all walks of life. Every sector relies on the internet for sharing information through internal and external mode of communication for its visibility. The educational institutions invest largely on knowledge resources which are maintained in the libraries depending upon the availability of budget. Every institution does not get equal amount of budget through which the libraries can acquire all the resources required for the stakeholders and fulfill them sufficiently. To eliminate this barrier and discrimination, the publishers and the knowledge creating institutions came out with open access policy to make the scholarly literature accessible freely through Internet. Though the scholarly literature is accessible online freely, many studies reported that the utilization of these resources is not up to the expectations. Therefore, it was intended to assess the level of awareness and extent of utilization of open access resources for teaching-learning and research purposes.

\section{LITERATURE REVIEW}

Based on the literature review, it was found that only a very limited number of studies particularly on open access resources have been conducted. Therefore, a few studies related to this area, are summarized below:

Isibika (2018) revealed that a considerable number of participants were moderately aware of the library subscribed e-resources. And the major constraints identified among the respondents in utilizing such resources were unstable network connectivity and lack of searching skills. In view of these constraints, the study suggested the library to improve the network connectivity with high bandwidth and to have library users with intensive training on information searching skills in order to promote the utilization of these subscribed e-resources optimally. Bosah (2017) found that the participants were quite aware of the "green" and "gold" routes and at the same time they were not familiarized with the "diamond" route. Ahmed (2017); Mahadevamurthy and Adithyakumari (2015) found that most of the participants indicated that they used online resources on a daily basis and also use of ejournals was reported as the central type of interest by students in both the universities. Interestingly, it was found that use of free electronic resources was most popular overall among the respondents. 
Sun et al (2017) revealed that "an individual's Chinese Universities of link sharing tools was determined by his or her continued use intention directly and subjective norm indirectly. Users' satisfaction on link sharing tools was the main factor affecting the continuance intention. Individuals' motivation needs such as cognitive needs, personal integrative needs, and social integrative needs and as well." In addition, Alhabash et al (2017) showed that majority of the participants spent most of their time daily on Instagram, followed by Snapchat, Facebook, and Twitter. They also indicated the highest use intensity for Snapchat and Instagram (nearly equally), followed by Facebook and Twitter.

Duy and Vaughan (2015) revealed that "vendor-provided electronic resource usage statistics were not currently standardized across vendors. This study investigated the feasibility of using locally collected data to check the reliability of vendor-provided data. The study found that the two types of data correlate well in terms of use patterns, but that actual usage values differ for many products." Malone and Videon (2015) conducted a comparative analysis on the instructions given by the information providers and the rate of utilization of electronic resources. It was found that interestingly the study proved an increased usage of electronic resources due to the instructions.

\section{OBJECTIVES OF THE STUDY}

The study was carried out with the following primary objectives:

1. To identify the demographic profile of the respondents;

2. To assess the level of awareness on the open access resources among the students of engineering colleges in Telangana state based on demographic variables;

3. To compare the level of awareness with the extent of utilization of open access resources by the respondents;

4. To examine the purpose of using open access resources by the respondents; and

5. To identify the problems associated with the use of open access resources by the respondents.

\section{RESEARCH DESIGN}

The region considered for the study is Telangana State. The research is based on the students studying in the engineering colleges. Out of 37 top ranked engineering institutions, only 6 were randomly selected for the study. Since, the commonalities were essentially required for comparative analysis of the respondents from same branches, six institutions, which offer the courses on Civil, Mechanical, EEE, ECE, and CSE, were identified. Thus, the list of colleges undertaken for the study is given below:

1. Guru Nanak Institutions Technical Campus, Secunderabad (GNITCS)

2. Maturi Venkata Subba Rao Engineering College, Hyderabad (MVSRECH)

3. National Institute of Technology Warangal (NITW)

4. CVR College of Engineering, Hyderabad (CVRCEH)

5. University College of Engineering, Osmania University, Hyderabad (UCEOUH)

6. Chaitanya Bharathi Institute of Technology, Hyderabad (CBITH)

A structured questionnaire was employed to collect the data. Simple random sampling was adopted for collecting opinion of the engineering students on the awareness and use of open access resources for teaching-learning and research. The target population constitutes only UG and PG students studying in engineering colleges in Telangana State. A total of 900 questionnaires were distributed. Of which, 766 questionnaires duly filled in were received 
back with a response rate of 85.11 (Table 1). The questionnaires were self-administered. Hereafter, abbreviations of the colleges will be used throughout the paper.

\section{RESULTS AND DISCUSSION}

Table 1 presents the profile of the respondents along with the response rate from six engineering institutions selected for the study. It was found that out of six engineering institutions, GNITCS ranks first in order by reporting 86.67 percentage rate of response followed by MVSRECH (86\%), NITW (85.33\%), CVRCEH (84.67\%), UCEOUH (84\%), and CBITH (84\%). The overall response rate comes to 85.11 percent.

Table 1 Profile of the Respondents

\begin{tabular}{|c|c|c|c|c|}
\hline $\begin{array}{l}\text { Sl. } \\
\text { No. }\end{array}$ & Institution & $\begin{array}{c}\text { Questionnaires } \\
\text { Distributed }\end{array}$ & $\begin{array}{c}\text { Questionnaires } \\
\text { Received }\end{array}$ & Response Rate \\
\hline 1 & GNITCS & 150 & 130 & 86.67 \\
\hline 2 & MVSRECH & 150 & 129 & 86.00 \\
\hline 3 & NITW & 150 & 128 & 85.33 \\
\hline 4 & CVRCEH & 150 & 127 & 84.67 \\
\hline 5 & UCEOUH & 150 & 126 & 84.00 \\
\hline 6 & $\mathrm{CBITH}$ & 150 & 126 & 84.00 \\
\hline \multicolumn{2}{|r|}{ Total } & 900 & 766 & 85.11 \\
\hline
\end{tabular}

\subsection{Respondents by Gender}

Table 2 highlights the gender wise distribution of the respondents from six engineering institutions in Telangana state. The result indicates that out of 766 total respondents, 441 $(57.57 \%)$ are male and $325(42.43 \%)$ respondents are female. The institution wise break up revealed that out of 441 male respondents, $84(19.05 \%)$ are from NITW which ranks first in order followed by MVSRECH (17.23\%), UCEOUH (16.10\%), GNITCS (15.42\%), CVRCEH $(13.38 \%)$, and CBITH $(18.82 \%)$. On the other hand, out of 325 female respondents, 68 $(19.05 \%)$ are from CVRCEH which ranks first in order followed by GNITCS (19.08\%), UCEOUH (16.92\%), MVSRECH (16.31\%), NITW (13.54\%), and CBITH (13.23\%)

Table 2 Gender wise distribution of the respondents

\begin{tabular}{|c|c|c|c|c|c|c|}
\hline Institution & Male & \% & Female & \% & Total & \% \\
\hline GNITCS & 68 & 15.42 & 62 & 19.08 & 130 & 16.97 \\
\hline MVSRECH & 76 & 17.23 & 53 & 16.31 & 129 & 16.84 \\
\hline NITW & 84 & 19.05 & 44 & 13.54 & 128 & 16.71 \\
\hline CVRCEH & 59 & 13.38 & 68 & 20.92 & 127 & 16.58 \\
\hline UCEOUH & 71 & 16.10 & 55 & 16.92 & 126 & 16.45 \\
\hline CBITH & 83 & 18.82 & 43 & 13.23 & 126 & 16.45 \\
\hline Total & 441 & 100 & 325 & 100 & 766 & 100 \\
\hline$\%$ & 57.57 & & 42.43 & & 100 & \\
\hline
\end{tabular}

\subsection{Age wise distribution of the respondents}

Table 3 indicates the age wise distribution of the respondents. It was found that out of 766 respondents, 324 respondents which records 42.3 percent fall under the age group of 21-24 years followed by 222 (28.98\%) respondents who fall under the age group of 17-20 years; 158 (20.63\%) respondents who fall under the age group of 25-28 years; 37 (4.83\%) respondents who fall under the age group of 29-32 years; and 25 (3.26\%) respondents who fall under the age group of 33 and above years. 
Jagadish, B and Anjaiah, M

Table 3 Distribution of the respondents by Age Group

\begin{tabular}{|c|c|c|c|c|c|c|}
\hline Institution & $\mathbf{1 7 - 2 0}$ & $\mathbf{2 1 - 2 4}$ & $\mathbf{2 5 - 2 8}$ & $\mathbf{2 9 - 3 2}$ & $\mathbf{3 3}$ and Above & Total \\
\hline GNITCS & $31(13.96)$ & $51(15.74)$ & $32(20.25)$ & $12(32.43)$ & $4(16)$ & $130(16.97)$ \\
\hline MVSRECH & $39(17.57)$ & $47(14.51)$ & $29(18.35)$ & $8(21.62)$ & $6(24)$ & $129(16.84)$ \\
\hline NITW & $49(22.07)$ & $54(16.67)$ & $14(8.86)$ & $6(16.22)$ & $5(20)$ & $128(16.71)$ \\
\hline CVRCEH & $46(20.72)$ & $48(14.81)$ & $26(16.46)$ & $4(10.81)$ & $3(12)$ & $127(16.58)$ \\
\hline UCEOUH & $28(12.61)$ & $60(18.52)$ & $31(19.62)$ & $4(10.81$ & $3(12)$ & $126(16.45)$ \\
\hline CBITH & $29(13.06)$ & $64(19.75)$ & $26(16.46)$ & $3(8.11)$ & $4(16)$ & $126(16.45)$ \\
\hline Total & $222(28.98)$ & $324(42.3)$ & $158(20.63)$ & $37(4.83)$ & $25(3.26)$ & $766(100)$ \\
\hline
\end{tabular}

\subsection{Respondents by Branch of Engineering}

Based on the commonalities in terms of disciplines which were identified among six institutions, the respondents were distributed by branch of engineering to which they belong. As shown in Table 4, the result reports that out of 766 total respondents, 233 (30.42\%) are from CSE department followed by 189(24.67\%) are from ECE; 130(16.97\%) are from EEE; $119(15.54 \%)$ are from Mechanical; and 95(12.4\%) are from Civil Engineering department.

Table 4 Distribution of respondents by branch of engineering

\begin{tabular}{|c|c|c|c|c|c|c|}
\hline Institution & Civil & Mechanical & EEE & ECE & CSE & Total \\
\hline GNITCS & $18(18.95)$ & $21(17.65)$ & $26(20)$ & $26(13.76)$ & $39(16.74)$ & $130(16.97)$ \\
\hline MVSRECH & $16(16.84)$ & $17(14.29)$ & $19(14.62)$ & $36(19.05)$ & $41(17.6)$ & $129(16.84)$ \\
\hline NITW & $16(16.84)$ & $19(15.97)$ & $23(17.69)$ & $33(17.46)$ & $37(15.88)$ & $128(16.71)$ \\
\hline CVRCEH & $17(17.89)$ & $22(18.49)$ & $21(16.15)$ & $29(15.34)$ & $38(16.31)$ & $127(16.58)$ \\
\hline UCEOUH & $15(15.79)$ & $21(17.65)$ & $17(13.08)$ & $32(16.93)$ & $41(17.6)$ & $126(16.45)$ \\
\hline CBITH & $13(13.68)$ & $19(15.97)$ & $24(18.46)$ & $33(17.46)$ & $37(15.88)$ & $126(16.45)$ \\
\hline Total & $95(12.4)$ & $119(15.54)$ & $130(16.97)$ & $189(24.67)$ & $233(30.42)$ & $766(100)$ \\
\hline
\end{tabular}

\subsection{Awareness Vs. Use of Open Access Resources by Institution}

The extent of use of open access resources among engineering students of various institutions from Telangana state was assessed. As shown in Table 5.15, the overall scenario revealed that out of various open access resources of which the respondents were aware, E-Journals were found to be the most used resource among the engineering students of the institutions under study by sharing 92.03 percentage followed by Academic Social Networking Sites (90.3\%), Online Reference Tools (89.39\%), E-Books (89.37\%), Online Tutorials (84.98\%), Open Courseware (78.78\%), Electronic Theses \& Dissertations (78.41\%), Subject Gateways (77.96\%), Online Directories (74.07\%), Institutional Repositories (69.62\%), Open Access Digital Libraries (68.52\%), Plagiarism Detection Tools (49.6\%), Journal Selection Tools (48.85\%), Reference Management Tools (47.51\%), and Manuscript Editing Tools (47.14\%).

With regard to institution wise level of usage of open access resources, 94.74 percent of the respondents from GNITCS made use of Academic Social Networking Sites which ranks first in order followed by Online Tutorials (91.36\%), E-Journals (90.32\%), E-Books (88.24\%), Online Lecture Videos (82.20\%), Theses \& Dissertations (79.66\%), Institutional Repositories (79.25\%), Open Courseware (79.17\%), Subject Gateways (78.76\%), Online Directories (74.42\%), Open Access Digital Libraries (72.82\%), Plagiarism Detection Tools (64.86\%), Journal Selection Tools (53.97\%), Manuscript Editing Tools (50.65\%), and Reference Management Tools (48.31\%).

It was found that 94.87 percent of the respondents from MVSRECH made use of EJournals which ranks first in order followed by Academic Social Networking Sites (90.10\%), E-Books (88.98\%), Online Tutorials (87.39\%), Theses \& Dissertations (86.61\%), Subject Gateways (83.74\%), Online Lecture Videos (83.61\%), Open Courseware (74.55\%), Institutional Repositories (73.45\%), Online Directories (69.57\%), Open Access Digital 
Libraries (66.67\%), Journal Selection Tools (52.78\%), Plagiarism Detection Tools (46.15\%), Manuscript Editing Tools (43.30\%), and Reference Management Tools (39.09\%).

With regard to NITW, 96.72 percent of the respondents made use of E-Journals which ranks first in order followed by Online Lecture Videos (95.93\%), E-Books $(91.06 \%)$, Academic Social Networking Sites (89.47\%), Online Tutorials (86.32\%), Theses \& Dissertations (79.67\%), Subject Gateways (77.05\%), Institutional Repositories $(74.17 \%)$, Open Courseware (69.42\%), Online Directories (60.38\%), Open Access Digital Libraries (57.14\%), Journal Selection Tools (50.88\%), Manuscript Editing Tools (46.02\%), Reference Management Tools (42.24\%), and Plagiarism Detection Tools (41.18\%).

Table 5 Distribution of Awareness Vs. Use of OAR by Institution

\begin{tabular}{|c|c|c|c|c|c|c|c|c|c|c|c|c|c|c|c|c|}
\hline $\begin{array}{c}\text { Institutio } \\
\text { n }\end{array}$ & Status & 1 & 2 & 3 & 4 & 5 & 6 & 7 & 8 & 9 & 10 & 11 & 12 & 13 & 14 & 15 \\
\hline \multirow{3}{*}{ GNITCS } & $\begin{array}{c}\text { Awaren } \\
\text { ess }\end{array}$ & 96 & 119 & 106 & 124 & 118 & 81 & 103 & 118 & 89 & 74 & 63 & 77 & 43 & 76 & 113 \\
\hline & Use & 76 & 105 & 84 & 112 & 94 & 74 & 75 & 97 & 43 & 48 & 34 & 39 & 32 & 72 & 89 \\
\hline & $\%$ & $\begin{array}{c}79.1 \\
7 \\
\end{array}$ & $\begin{array}{c}88.2 \\
4 \\
\end{array}$ & $\begin{array}{c}79.2 \\
5 \\
\end{array}$ & $\begin{array}{c}90.3 \\
2 \\
\end{array}$ & $\begin{array}{c}79.6 \\
6 \\
\end{array}$ & $\begin{array}{c}91.3 \\
6 \\
\end{array}$ & $\begin{array}{c}72.8 \\
2 \\
\end{array}$ & $\begin{array}{c}82.2 \\
0 \\
\end{array}$ & $\begin{array}{c}48.3 \\
1 \\
\end{array}$ & $\begin{array}{c}64.8 \\
6 \\
\end{array}$ & $\begin{array}{c}53.9 \\
7 \\
\end{array}$ & $\begin{array}{c}50.6 \\
5 \\
\end{array}$ & $\begin{array}{c}74.4 \\
2 \\
\end{array}$ & $\begin{array}{c}94.7 \\
4 \\
\end{array}$ & $\begin{array}{c}78.7 \\
6\end{array}$ \\
\hline \multirow{3}{*}{$\begin{array}{c}\text { MVSRE } \\
\mathrm{CH}\end{array}$} & $\begin{array}{c}\text { Awaren } \\
\text { ess }\end{array}$ & 110 & 118 & 113 & 117 & 112 & 111 & 117 & 122 & 110 & 104 & 108 & 97 & 92 & 101 & 123 \\
\hline & Use & 82 & 105 & 83 & 111 & 97 & 97 & 78 & 102 & 43 & 48 & 57 & 42 & 64 & 91 & 103 \\
\hline & $\%$ & $\begin{array}{c}74.5 \\
5\end{array}$ & $\begin{array}{c}88.9 \\
8\end{array}$ & $\begin{array}{c}73.4 \\
5\end{array}$ & $\begin{array}{c}94.8 \\
7\end{array}$ & $\begin{array}{c}86.6 \\
1\end{array}$ & $\begin{array}{c}87.3 \\
9\end{array}$ & $\begin{array}{c}66.6 \\
7\end{array}$ & $\begin{array}{c}83.6 \\
1\end{array}$ & $\begin{array}{c}39.0 \\
9\end{array}$ & $\begin{array}{c}46.1 \\
5\end{array}$ & $\begin{array}{c}52.7 \\
8\end{array}$ & $\begin{array}{c}43.3 \\
0\end{array}$ & $\begin{array}{c}69.5 \\
7\end{array}$ & $\begin{array}{c}90.1 \\
0\end{array}$ & $\begin{array}{c}83.7 \\
4\end{array}$ \\
\hline \multirow{3}{*}{ NITW } & $\begin{array}{c}\text { Awaren } \\
\text { ess }\end{array}$ & 121 & 123 & 120 & 122 & 123 & 117 & 119 & 123 & 116 & 119 & 114 & 113 & 106 & 114 & 122 \\
\hline & Use & 84 & 112 & 89 & 118 & 98 & 101 & 68 & 118 & 49 & 49 & 58 & 52 & 64 & 102 & 94 \\
\hline & $\%$ & $\begin{array}{c}69.4 \\
2 \\
\end{array}$ & $\begin{array}{c}91.0 \\
6 \\
\end{array}$ & $\begin{array}{c}74.1 \\
7 \\
\end{array}$ & $\begin{array}{c}96.7 \\
2 \\
\end{array}$ & $\begin{array}{c}79.6 \\
7 \\
\end{array}$ & $\begin{array}{c}86.3 \\
2 \\
\end{array}$ & $\begin{array}{c}57.1 \\
4 \\
\end{array}$ & $\begin{array}{c}95.9 \\
3 \\
\end{array}$ & $\begin{array}{c}42.2 \\
4 \\
\end{array}$ & $\begin{array}{c}41.1 \\
8\end{array}$ & $\begin{array}{c}50.8 \\
8 \\
\end{array}$ & $\begin{array}{c}46.0 \\
2 \\
\end{array}$ & $\begin{array}{c}60.3 \\
8 \\
\end{array}$ & $\begin{array}{c}89.4 \\
7 \\
\end{array}$ & $\begin{array}{c}77.0 \\
5 \\
\end{array}$ \\
\hline \multirow{3}{*}{$\begin{array}{c}\text { CVRCE } \\
\mathrm{H}\end{array}$} & $\begin{array}{c}\text { Awaren } \\
\text { ess }\end{array}$ & 101 & 113 & 118 & 119 & 115 & 116 & 118 & 121 & 106 & 90 & 102 & 80 & 56 & 89 & 108 \\
\hline & Use & 83 & 96 & 85 & 108 & 87 & 86 & 79 & 111 & 59 & 38 & 49 & 39 & 43 & 81 & 82 \\
\hline & $\%$ & $\begin{array}{c}82.1 \\
8 \\
\end{array}$ & $\begin{array}{c}84.9 \\
6 \\
\end{array}$ & $\begin{array}{c}72.0 \\
3 \\
\end{array}$ & $\begin{array}{c}90.7 \\
6 \\
\end{array}$ & $\begin{array}{c}75.6 \\
5 \\
\end{array}$ & $\begin{array}{c}74.1 \\
4 \\
\end{array}$ & $\begin{array}{c}66.9 \\
5 \\
\end{array}$ & $\begin{array}{c}91.7 \\
4 \\
\end{array}$ & $\begin{array}{c}55.6 \\
6 \\
\end{array}$ & $\begin{array}{c}42.2 \\
2 \\
\end{array}$ & $\begin{array}{c}48.0 \\
4\end{array}$ & $\begin{array}{c}48.7 \\
5 \\
\end{array}$ & $\begin{array}{c}76.7 \\
9 \\
\end{array}$ & $\begin{array}{c}91.0 \\
1 \\
\end{array}$ & $\begin{array}{c}75.9 \\
3 \\
\end{array}$ \\
\hline \multirow{3}{*}{$\begin{array}{c}\text { UCEOU } \\
\text { H }\end{array}$} & $\begin{array}{c}\text { Awaren } \\
\text { ess }\end{array}$ & 114 & 115 & 118 & 117 & 115 & 107 & 112 & 119 & 110 & 104 & 115 & 101 & 98 & 99 & 113 \\
\hline & Use & 89 & 102 & 68 & 103 & 86 & 92 & 82 & 105 & 62 & 53 & 52 & 44 & 79 & 89 & 89 \\
\hline & $\%$ & $\begin{array}{c}78.0 \\
7 \\
\end{array}$ & $\begin{array}{c}88.7 \\
0 \\
\end{array}$ & $\begin{array}{c}57.6 \\
3 \\
\end{array}$ & $\begin{array}{c}88.0 \\
3 \\
\end{array}$ & $\begin{array}{c}74.7 \\
8 \\
\end{array}$ & $\begin{array}{c}85.9 \\
8 \\
\end{array}$ & $\begin{array}{c}73.2 \\
1 \\
\end{array}$ & $\begin{array}{c}88.2 \\
4 \\
\end{array}$ & $\begin{array}{c}56.3 \\
6 \\
\end{array}$ & $\begin{array}{c}50.9 \\
6 \\
\end{array}$ & $\begin{array}{c}45.2 \\
2 \\
\end{array}$ & $\begin{array}{c}43.5 \\
6 \\
\end{array}$ & $\begin{array}{c}80.6 \\
1 \\
\end{array}$ & $\begin{array}{c}89.9 \\
0 \\
\end{array}$ & $\begin{array}{c}78.7 \\
6 \\
\end{array}$ \\
\hline \multirow{3}{*}{ CBITH } & $\begin{array}{c}\text { Awaren } \\
\text { ess }\end{array}$ & 103 & 105 & 116 & 117 & 112 & 111 & 117 & 112 & 113 & 90 & 109 & 91 & 75 & 67 & 102 \\
\hline & Use & 92 & 99 & 71 & 107 & 83 & 94 & 87 & 106 & 49 & 47 & 46 & 46 & 62 & 58 & 75 \\
\hline & $\%$ & $\begin{array}{c}89.3 \\
2 \\
\end{array}$ & $\begin{array}{c}94.2 \\
9 \\
\end{array}$ & $\begin{array}{c}61.2 \\
1 \\
\end{array}$ & $\begin{array}{c}91.4 \\
5 \\
\end{array}$ & $\begin{array}{c}74.1 \\
1 \\
\end{array}$ & $\begin{array}{c}84.6 \\
8 \\
\end{array}$ & $\begin{array}{c}74.3 \\
6 \\
\end{array}$ & $\begin{array}{c}94.6 \\
4 \\
\end{array}$ & $\begin{array}{c}43.3 \\
6 \\
\end{array}$ & $\begin{array}{c}52.2 \\
2\end{array}$ & $\begin{array}{c}42.2 \\
0\end{array}$ & $\begin{array}{c}50.5 \\
5 \\
\end{array}$ & $\begin{array}{c}82.6 \\
7 \\
\end{array}$ & $\begin{array}{c}86.5 \\
7 \\
\end{array}$ & $\begin{array}{c}73.5 \\
3\end{array}$ \\
\hline \multicolumn{2}{|c|}{ Overall \% } & $\begin{array}{c}78.7 \\
8 \\
\end{array}$ & $\begin{array}{c}89.3 \\
7 \\
\end{array}$ & $\begin{array}{c}69.6 \\
2 \\
\end{array}$ & $\begin{array}{c}92.0 \\
3 \\
\end{array}$ & $\begin{array}{c}78.4 \\
1 \\
\end{array}$ & $\begin{array}{c}84.9 \\
8 \\
\end{array}$ & $\begin{array}{c}68.5 \\
2 \\
\end{array}$ & $\begin{array}{c}89.3 \\
9 \\
\end{array}$ & $\begin{array}{c}47.5 \\
1 \\
\end{array}$ & $\begin{array}{c}49.6 \\
0 \\
\end{array}$ & $\begin{array}{c}48.8 \\
5 \\
\end{array}$ & $\begin{array}{c}47.1 \\
4 \\
\end{array}$ & $\begin{array}{c}74.0 \\
7 \\
\end{array}$ & $\begin{array}{c}90.3 \\
0 \\
\end{array}$ & $\begin{array}{c}77.9 \\
6 \\
\end{array}$ \\
\hline
\end{tabular}

1=Open Courseware; 2=E-Books; 3=Institutional Repositories; 4=E-Journals; 5=Theses \& Dissertations; 6=Online Tutorials; 7=Open Access Digital Libraries; 8=Online Lecture Videos; 9=Reference Management Tools; 10=Plagiarism Detection Tools; 11=Journal Selection Tools; 12=Manuscript Editing Tools; 13=Online Directories; 14=Academic Social Networking Sites; $15=$ Subject Gateways

As far as CVRCEH is concerned, 91.74 percent of the respondents made use of Online Lecture Videos which ranks first in order followed by Academic Social Networking Sites (91.01\%), E-Journals (90.76\%), E-Books (84.96\%), Open Courseware (82.18\%), Online Directories (76.79\%), Subject Gateways (75.93\%), Theses \& Dissertations (75.65\%), Online Tutorials (74.14\%), Institutional Repositories (72.03\%), Open Access Digital Libraries 
(66.95\%), Reference Management Tools (55.66\%), Manuscript Editing Tools (48.75\%), Journal Selection Tools (48.04\%), and Plagiarism Detection Tools (42.22\%).

With regard to UCEOUH, 96.72 percent of the respondents made use of Academic Social Networking Sites which ranks first in order followed by E-Books (88.70\%), Online Lecture Videos (88.24\%), E-Journals (88.03\%), Online Tutorials $(85.98 \%)$, Online Directories (80.61\%), Subject Gateways (78.76\%), Open Courseware (78.07\%), Theses \& Dissertations (74.78\%), Open Access Digital Libraries (73.21\%), Institutional Repositories (57.63\%), Reference Management Tools (56.36\%), Plagiarism Detection Tools (50.96\%), Journal Selection Tools (45.22\%), and Manuscript Editing Tools (43.56\%).

It was found that 94.64 percent of the respondents from CBITH made use of Online Lecture Videos which ranks first in order followed by E-Books (94.29\%), E-Journals (91.45\%), Open Courseware (89.32\%), Academic Social Networking Sites (86.57\%), Online Tutorials (84.68\%), Online Directories (82.67\%), Open Access Digital Libraries (74.36\%), Theses \& Dissertations (74.11\%), Subject Gateways (73.53\%), Institutional Repositories (61.21\%), Plagiarism Detection Tools (52.22\%), Manuscript Editing Tools (50.55\%), Reference Management Tools (43.36\%), and Journal Selection Tools (42.20\%).

\subsection{Awareness vs. Use of Open Access Resources by Gender}

Table 6 highlights the gender wise awareness versus use of open access resources among the engineering students of selected engineering institutions in Telangana state. It was found that 98.07 percent of the male respondents made use of Academic Social Networking Sites which ranks first in order followed by E-Journals (96.23\%), E-Books (94.50\%), Online Tutorials (94.24\%), Theses \& Dissertations (93.22\%), Online Lecture Videos (88.12\%), Open Access Digital Libraries (83.04\%), Institutional Repositories (81.89\%), Open Courseware (78.80\%), Reference Management Tools (69.64\%), Subject Gateways (66.41\%), Online Directories (66.08\%), Plagiarism Detection Tools (58.59\%), Manuscript Editing Tools (43.21\%), and Journal Selection Tools (41.13\%).

On the other hand, 97.56 percent of the female respondents made use of Academic Social Networking Sites which ranks first in order followed by E-Books (97.36\%), E-Journals (97.30\%), Online Tutorials (95.94\%), Theses \& Dissertations (89.15\%), Online Lecture Videos (87.97\%), Open Courseware (82.57\%), Institutional Repositories (79.73\%), Open Access Digital Libraries (75.83\%), Online Directories (67.78\%), Subject Gateways (62.85\%), Plagiarism Detection Tools (62.03\%), Reference Management Tools (52.91\%), Manuscript Editing Tools (41.31\%), and Journal Selection Tools (38.92\%).

Overall, it was found that 97.82 percent of the total respondents made use of Academic Social Networking Sites which ranks first in order followed by E-Journals (96.76\%), E-Books (95.93\%), Online Tutorials (95.09\%), Theses \& Dissertations (91.18\%), Online Lecture Videos (88.04\%), Institutional Repositories (80.81\%), Open Courseware (80.69\%), Open Access Digital Libraries (79.44\%), Online Directories (66.93\%), Subject Gateways (64.63\%), Reference Management Tools (61.27\%), Plagiarism Detection Tools (60.31\%), Manuscript Editing Tools (42.26\%), and Journal Selection Tools (40.02\%). 
Awareness and Use of Open Access Resources among the Students of Selected Engineering Institutions in Telangana State: A Survey

Table 6 Distribution of Awareness vs. Use of OAR by Gender

\begin{tabular}{|c|c|c|c|c|c|c|c|}
\hline \multirow{2}{*}{ OAR } & \multicolumn{3}{|c|}{ Male } & \multicolumn{3}{|c|}{ Female } & \multirow{2}{*}{$\%$ Average } \\
\hline & Awareness & Use & $\%$ & Awareness & Use & $\%$ & \\
\hline Open Courseware & 368 & 290 & 78.80 & 248 & 205 & 82.57 & 80.69 \\
\hline E-Books & 382 & 361 & 94.50 & 259 & 252 & 97.36 & 95.93 \\
\hline Institutional Repositories & 403 & 330 & 81.89 & 253 & 202 & 79.73 & 80.81 \\
\hline E-Journals & 424 & 408 & 96.23 & 289 & 281 & 97.30 & 96.76 \\
\hline Theses \& Dissertations & 398 & 371 & 93.22 & 264 & 235 & 89.15 & 91.18 \\
\hline Online Tutorials & 330 & 311 & 94.24 & 283 & 272 & 95.94 & 95.09 \\
\hline $\begin{array}{c}\text { Open Access Digital } \\
\text { Libraries }\end{array}$ & 336 & 279 & 83.04 & 221 & 168 & 75.83 & 79.44 \\
\hline Online Lecture Videos & 345 & 304 & 88.12 & 238 & 209 & 87.97 & 88.04 \\
\hline $\begin{array}{c}\text { Reference Management } \\
\text { Tools }\end{array}$ & 303 & 211 & 69.64 & 259 & 137 & 52.91 & 61.27 \\
\hline $\begin{array}{c}\text { Plagiarism Detection } \\
\text { Tools } \\
\end{array}$ & 297 & 174 & 58.59 & 248 & 154 & 62.03 & 60.31 \\
\hline Journal Selection Tools & 248 & 102 & 41.13 & 267 & 104 & 38.92 & 40.02 \\
\hline Manuscript Editing Tools & 287 & 124 & 43.21 & 201 & 83 & 41.31 & 42.26 \\
\hline Online Directories & 286 & 189 & 66.08 & 236 & 160 & 67.78 & 66.93 \\
\hline $\begin{array}{l}\text { Academic Social } \\
\text { Networking Sites }\end{array}$ & 311 & 305 & 98.07 & 283 & 276 & 97.56 & 97.82 \\
\hline Subject Gateways & 387 & 257 & 66.41 & 261 & 164 & 62.85 & 64.63 \\
\hline
\end{tabular}

\subsection{Awareness vs. Use of Open Access Resources by Age}

Table 7 highlights the age wise awareness versus use of open access resources among the engineering students of selected engineering institutions in Telangana state. It was found that 93.09 percent of the respondents who fall under the age group of 17-20 made use of Academic Social Networking Sites which ranks first in order followed by Online Lecture Videos (91.68\%), Online Tutorials (90.85\%), E-Books (89.81\%), E-Journals (85.49\%), Subject Gateways (83.5\%), Institutional Repositories (80.65\%), Online Directories $(66.25 \%)$, Open Courseware (64.98\%), Open Access Digital Libraries (60.87\%), Theses \& Dissertations (40.61\%), Plagiarism Detection Tools (30.42\%), Journal Selection Tools (24.24\%), Manuscript Editing Tools (22.72\%), and Reference Management Tools (22.41\%).

With regard to the respondents who fall under the age group of 21-24, it was found that 96.21 percent of the respondents made use of E-Books which ranks first in order followed by Online Tutorials (95.7\%), Academic Social Networking Sites (93.83\%), Subject Gateways (93.73\%), E-Journals (92.43\%), Online Lecture Videos (91.05\%), Institutional Repositories (88.04\%), Open Courseware (77.25\%), Open Access Digital Libraries $\quad$ (76.55\%), Online Directories (72.21\%), Theses \& Dissertations (65.52\%), Journal Selection Tools $(51.56 \%)$, Manuscript Editing Tools (50.98\%), Reference Management Tools (43.63\%), and Plagiarism Detection Tools $(41.07 \%)$.

In respect of the respondents who fall under the age group of 25-28, it was found that 95.95 percent of the respondents made use of E-Books which ranks first in order followed by Academic Social Networking Sites (93.08\%), E-Journals (91.78\%), Online Tutorials (89.46\%), Online Lecture Videos (88.76\%), Open Courseware (85.5\%), Open Access Digital Libraries (85.47\%), Institutional Repositories (85.08\%), Subject Gateways (78.09\%), Online Directories (69.5\%), Theses \& Dissertations (68.43\%), Journal Selection Tools (59.61\%), Manuscript Editing Tools (52.47\%), Reference Management Tools (48.92\%), and Plagiarism Detection Tools (46.91\%).

As far as the respondents who fall under the age group of 29-32 are concerned, it was found that 95.48 percent of the respondents made use of Online Tutorials which ranks first in order followed by Online Lecture Videos (94.81\%), E-Books (91.94\%), Academic Social 
Networking Sites (90.32\%), E-Journals (89.29\%), Subject Gateways (87.45\%), Reference Management Tools (82.22\%), Institutional Repositories (81.66\%), Open Courseware (76.39\%), Journal Selection Tools (74\%), Theses \& Dissertations (71.89\%), Open Access Digital Libraries (66.84\%), Online Directories (65.78\%), Plagiarism Detection Tools (61.13\%), Manuscript Editing Tools (52.42\%).

With regard to the respondents who fall under the age group of 33 and above, it was found that 96.01 percent of the respondents made use of E-Journals which ranks first in order followed by Theses \& Dissertations (93.38\%), Open Access Digital Libraries (90.48\%), Online Tutorials (85.13\%), Online Directories (85.13\%), Institutional Repositories (84.49\%), Academic Social Networking Sites (84.49\%), Open Courseware (80.65\%), Journal Selection Tools (80.65\%), Subject Gateways (80.65\%), Online Lecture Videos (77.14\%), Reference Management Tools (76.61\%), E-Books (73.63\%), Manuscript Editing Tools (62.72\%), and Plagiarism Detection Tools (56.93\%).

Overall, it was found that 91.32 percent of the total respondents made use of Online Tutorials which ranks first in order followed by E-Journals (91\%), Academic Social Networking Sites (90.96\%), E-Books (89.51\%), Online Lecture Videos (88.69\%), Subject Gateways (84.68\%), Institutional Repositories (83.98\%), Open Courseware (76.95\%), Open Access Digital Libraries (76.04\%), Online Directories (71.77\%), Theses \& Dissertations (67.97\%), Journal Selection Tools (58.01\%), Reference Management Tools (54.76\%), Manuscript Editing Tools (48.26\%), and Plagiarism Detection Tools (47.29\%).

Table 7 Distribution of Awareness vs. Use of OAR by Age Group

\begin{tabular}{|c|c|c|c|c|c|c|c|c|c|c|c|c|c|c|c|c|}
\hline $\begin{array}{c}\text { Age } \\
\text { Group }\end{array}$ & Status & 1 & 2 & 3 & 4 & 5 & 6 & 7 & 8 & 9 & 10 & 11 & 12 & 13 & 14 & 15 \\
\hline \multirow[t]{3}{*}{$17-20$} & $\begin{array}{c}\text { Awaren } \\
\text { ess }\end{array}$ & 91 & 163 & 119 & 182 & 148 & 159 & 129 & 175 & 106 & 94 & 52 & 96 & 169 & 214 & 119 \\
\hline & Use & 59 & 146 & 96 & 156 & 60 & 144 & 79 & 160 & 24 & 29 & 13 & 22 & 112 & 199 & 99 \\
\hline & $\%$ & $\begin{array}{c}64.9 \\
8 \\
\end{array}$ & $\begin{array}{c}89.8 \\
1 \\
\end{array}$ & $\begin{array}{c}80.6 \\
5 \\
\end{array}$ & $\begin{array}{c}85.4 \\
9 \\
\end{array}$ & $\begin{array}{c}40.6 \\
1 \\
\end{array}$ & $\begin{array}{c}90.8 \\
5 \\
\end{array}$ & $\begin{array}{c}60.8 \\
7 \\
\end{array}$ & $\begin{array}{c}91.6 \\
8 \\
\end{array}$ & $\begin{array}{c}22.4 \\
1 \\
\end{array}$ & $\begin{array}{c}30.4 \\
2 \\
\end{array}$ & $\begin{array}{c}24.2 \\
4\end{array}$ & $\begin{array}{c}22.7 \\
2 \\
\end{array}$ & $\begin{array}{c}66.2 \\
5 \\
\end{array}$ & $\begin{array}{c}93.0 \\
9 \\
\end{array}$ & $\begin{array}{c}83.5 \\
0 \\
\end{array}$ \\
\hline \multirow[t]{3}{*}{$21-24$} & $\begin{array}{c}\text { Awaren } \\
\text { ess }\end{array}$ & 255 & 247 & 243 & 251 & 255 & 243 & 233 & 261 & 185 & 224 & 231 & 217 & 222 & 302 & 218 \\
\hline & Use & 197 & 238 & 214 & 232 & 167 & 233 & 178 & 238 & 81 & 92 & 119 & 111 & 160 & 283 & 204 \\
\hline & $\%$ & $\begin{array}{c}77.2 \\
5 \\
\end{array}$ & $\begin{array}{c}96.2 \\
1 \\
\end{array}$ & $\begin{array}{c}88.0 \\
4 \\
\end{array}$ & $\begin{array}{c}92.4 \\
3 \\
\end{array}$ & $\begin{array}{c}65.5 \\
2 \\
\end{array}$ & $\begin{array}{c}95.7 \\
0 \\
\end{array}$ & $\begin{array}{c}76.5 \\
5 \\
\end{array}$ & $\begin{array}{c}91.0 \\
5 \\
\end{array}$ & $\begin{array}{c}43.6 \\
3 \\
\end{array}$ & $\begin{array}{c}41.0 \\
7 \\
\end{array}$ & $\begin{array}{c}51.5 \\
6 \\
\end{array}$ & $\begin{array}{c}50.9 \\
8 \\
\end{array}$ & $\begin{array}{c}72.2 \\
1 \\
\end{array}$ & $\begin{array}{c}93.8 \\
3 \\
\end{array}$ & $\begin{array}{c}93.7 \\
3 \\
\end{array}$ \\
\hline \multirow[t]{3}{*}{$25-28$} & $\begin{array}{c}\text { Awaren } \\
\text { ess }\end{array}$ & 104 & 116 & 103 & 138 & 129 & 133 & 119 & 129 & 102 & 45 & 88 & 89 & 81 & 145 & 77 \\
\hline & Use & 89 & 111 & 88 & 127 & 88 & 119 & 102 & 115 & 50 & 21 & 52 & 47 & 56 & 135 & 60 \\
\hline & $\%$ & $\begin{array}{c}85.5 \\
0\end{array}$ & $\begin{array}{c}95.9 \\
5\end{array}$ & $\begin{array}{c}85.0 \\
8\end{array}$ & $\begin{array}{c}91.7 \\
8\end{array}$ & $\begin{array}{c}68.4 \\
3\end{array}$ & $\begin{array}{c}89.4 \\
6\end{array}$ & $\begin{array}{c}85.4 \\
7\end{array}$ & $\begin{array}{c}88.7 \\
6\end{array}$ & $\begin{array}{c}48.9 \\
2\end{array}$ & $\begin{array}{c}46.9 \\
1\end{array}$ & $\begin{array}{c}59.6 \\
1\end{array}$ & $\begin{array}{c}52.4 \\
7\end{array}$ & $\begin{array}{c}69.5 \\
0\end{array}$ & $\begin{array}{c}93.0 \\
8\end{array}$ & $\begin{array}{c}78.0 \\
9\end{array}$ \\
\hline \multirow[t]{3}{*}{$29-32$} & $\begin{array}{c}\text { Awaren } \\
\text { ess }\end{array}$ & 31 & 33 & 29 & 28 & 35 & 31 & 31 & 32 & 27 & 23 & 26 & 24 & 27 & 31 & 22 \\
\hline & Use & 24 & 30 & 24 & 25 & 25 & 30 & 21 & 30 & 22 & 14 & 19 & 13 & 18 & 28 & 19 \\
\hline & $\%$ & $\begin{array}{c}76.3 \\
9 \\
\end{array}$ & $\begin{array}{c}91.9 \\
4 \\
\end{array}$ & $\begin{array}{c}81.6 \\
6 \\
\end{array}$ & $\begin{array}{c}89.2 \\
9 \\
\end{array}$ & $\begin{array}{c}71.8 \\
9 \\
\end{array}$ & $\begin{array}{c}95.4 \\
8 \\
\end{array}$ & $\begin{array}{c}66.8 \\
4 \\
\end{array}$ & $\begin{array}{c}94.8 \\
1 \\
\end{array}$ & $\begin{array}{c}82.2 \\
2 \\
\end{array}$ & $\begin{array}{c}61.1 \\
3 \\
\end{array}$ & $\begin{array}{c}74.0 \\
0 \\
\end{array}$ & $\begin{array}{c}52.4 \\
2 \\
\end{array}$ & $\begin{array}{c}65.7 \\
8 \\
\end{array}$ & $\begin{array}{c}90.3 \\
2 \\
\end{array}$ & $\begin{array}{c}87.4 \\
5 \\
\end{array}$ \\
\hline \multirow[t]{3}{*}{$\begin{array}{l}33 \text { and } \\
\text { Above }\end{array}$} & $\begin{array}{c}\text { Awaren } \\
\text { ess }\end{array}$ & 22 & 23 & 21 & 21 & 19 & 18 & 21 & 23 & 20 & 17 & 16 & 9 & 18 & 21 & 16 \\
\hline & Use & 18 & 17 & 18 & 20 & 18 & 15 & 19 & 18 & 15 & 10 & 13 & 6 & 15 & 18 & 13 \\
\hline & $\%$ & $\begin{array}{c}80.6 \\
5 \\
\end{array}$ & $\begin{array}{c}73.6 \\
3 \\
\end{array}$ & $\begin{array}{c}84.4 \\
9 \\
\end{array}$ & $\begin{array}{c}96.0 \\
1 \\
\end{array}$ & $\begin{array}{c}93.3 \\
8 \\
\end{array}$ & $\begin{array}{c}85.1 \\
3 \\
\end{array}$ & $\begin{array}{c}90.4 \\
8 \\
\end{array}$ & $\begin{array}{c}77.1 \\
4 \\
\end{array}$ & $\begin{array}{c}76.6 \\
1 \\
\end{array}$ & $\begin{array}{c}56.9 \\
3 \\
\end{array}$ & $\begin{array}{c}80.6 \\
5 \\
\end{array}$ & $\begin{array}{c}62.7 \\
2 \\
\end{array}$ & $\begin{array}{c}85.1 \\
3 \\
\end{array}$ & $\begin{array}{c}84.4 \\
9 \\
\end{array}$ & $\begin{array}{c}80.6 \\
5 \\
\end{array}$ \\
\hline \multicolumn{2}{|c|}{ Overall \% } & $\begin{array}{c}76.9 \\
5 \\
\end{array}$ & $\begin{array}{c}89.5 \\
1 \\
\end{array}$ & $\begin{array}{c}83.9 \\
8 \\
\end{array}$ & $\begin{array}{c}91.0 \\
0\end{array}$ & $\begin{array}{c}67.9 \\
7 \\
\end{array}$ & $\begin{array}{c}91.3 \\
2 \\
\end{array}$ & $\begin{array}{c}76.0 \\
4 \\
\end{array}$ & $\begin{array}{c}88.6 \\
9 \\
\end{array}$ & $\begin{array}{c}54.7 \\
6 \\
\end{array}$ & $\begin{array}{c}47.2 \\
9 \\
\end{array}$ & $\begin{array}{c}58.0 \\
1\end{array}$ & $\begin{array}{c}48.2 \\
6\end{array}$ & $\begin{array}{c}71.7 \\
7 \\
\end{array}$ & $\begin{array}{c}90.9 \\
6\end{array}$ & $\begin{array}{c}84.6 \\
8 \\
\end{array}$ \\
\hline
\end{tabular}

1=Open Courseware; 2=E-Books; 3=Institutional Repositories; 4=E-Journals; 5=Theses \& Dissertations; 6=Online Tutorials; 7=Open Access Digital Libraries; 8=Online Lecture Videos; 9=Reference Management Tools; 10=Plagiarism Detection Tools; 11=Journal Selection Tools; 12=Manuscript Editing Tools; 13=Online Directories; 14=Academic Social Networking Sites; 15=Subject Gateways 


\subsection{Purpose of using Open Access Resources by the respondents}

Table 8 visualizes different purposes of the respondents in making use of open access resources. The result indicates that out of ten different purposes mentioned, 'Preparing for curriculum based examinations' was found to be the primary purpose of using open access resources reporting 91.91 percent of the total respondents followed by 'To gather study related information' (83.29\%), 'For General Reading' (78.46\%), 'To keep up-to-date' (72.85\%), 'To prepare for competitive examinations' (70.37\%), 'To write research papers' (64.36\%), 'For Placement Details' (54.70\%), 'To share information' (43.21\%), 'To prepare for seminar, assignments, etc.' (42.69\%), and 'To repackage information' (22.45\%).

Table 8 Purpose of using OAR

\begin{tabular}{|c|c|c|c|}
\hline $\begin{array}{c}\text { S. } \\
\text { No. }\end{array}$ & Purpose & No. of Responses (N=766) & $\%$ \\
\hline 1 & To prepare for curriculum based examinations & 704 & 91.91 \\
\hline 2 & To gather study related information & 638 & 83.29 \\
\hline 3 & To prepare for seminar, assignments, etc. & 327 & 42.69 \\
\hline 4 & For General Reading & 601 & 78.46 \\
\hline 5 & To keep up-to-date & 558 & 72.85 \\
\hline 6 & To prepare for competitive examinations & 539 & 70.37 \\
\hline 7 & To write research papers & 493 & 64.36 \\
\hline 8 & For Placement Details & 419 & 54.70 \\
\hline 9 & To share information & 331 & 43.21 \\
\hline 10 & To repackage information & 172 & 22.45 \\
\hline
\end{tabular}

\subsection{Problems faced by the respondents while using open access resources}

It is quite practical that there will be a lot of variations among academic institutions in terms of infrastructure which includes learning resources, services, internet facility, etc. Although the open access resources are freely available, having convenient access to these resources without any hindrances depends on the sound infrastructure of an institution. In addition, the open access resources may themselves have their own merits and demerits. Therefore, it is worth studying the problems faced by the respondents either with the infrastructure or the resources themselves while using these resources. The emphasis has been laid on the institutional variations in the problems faced by the respondents.

As can be seen in Table 9, a list of 15 major problems was posed to the respondents for their opinion. It was found that out of various problems, the major problem faced by 86.01 percent of the respondents from GNITCS was 'Lack of Authenticity' followed by Lack of knowledge in identifying fake publishers/journals (85.22\%), Lack of subject coverage (81.51\%), Lack of Speed (71.22\%), Lack of Quality (67.49\%), Lack of Search skills (67.19\%), Non-availability of full text (63.09\%), Lack of Time (63.09\%), Inactive Links (61.01\%), Lack of up-to-date information (58.75\%), Incomplete bibliographic information (58.24\%), Slow and hidden feedback (55.27\%), Inadequate search facility (55.25\%), Lack of user friendliness (45.2\%), and Lack of Interest (42.09\%). 
Table 9 Distribution of the problems faced by the respondents while accessing open access resources

\begin{tabular}{|c|c|c|c|c|c|c|c|}
\hline Problems Faced & GNITCS & $\begin{array}{c}\text { MVSREC } \\
\text { H }\end{array}$ & NITW & CVRCEH & UCEOUH & CBITH & Average \\
\hline Lack of Authenticity & 86.01 & 87.32 & 91.31 & 84.77 & 74.43 & 73.85 & 82.95 \\
\hline Lack of Quality & 67.49 & 68.8 & 78.79 & 66.25 & 65.91 & 65.33 & 68.76 \\
\hline $\begin{array}{l}\text { Incomplete } \\
\text { bibliographic } \\
\text { information }\end{array}$ & 58.24 & 57.55 & 64.56 & 57 & 56.66 & 56.08 & 58.35 \\
\hline $\begin{array}{l}\text { Non-availability of } \\
\text { full text }\end{array}$ & 63.09 & 65.4 & 62.39 & 61.85 & 51.51 & 50.93 & 59.20 \\
\hline $\begin{array}{l}\text { Inadequate search } \\
\text { facility }\end{array}$ & 55.25 & 56.56 & 56.55 & 54.01 & 63.67 & 63.09 & 58.19 \\
\hline $\begin{array}{c}\text { Lack of subject } \\
\text { coverage }\end{array}$ & 81.51 & 81.79 & 84.81 & 80.27 & 69.93 & 69.35 & 77.94 \\
\hline Inactive Links & 61.01 & 62.32 & 58.43 & 59.77 & 48.43 & 47.85 & 56.30 \\
\hline $\begin{array}{l}\text { Lack of user } \\
\text { friendliness }\end{array}$ & 45.2 & 46.51 & 42.62 & 43.92 & 43.62 & 43.04 & 44.15 \\
\hline $\begin{array}{l}\text { Lack of up-to-date } \\
\text { information }\end{array}$ & 58.75 & 61 & 68.17 & 57.51 & 48.17 & 47.59 & 56.87 \\
\hline $\begin{array}{l}\text { Lack of knowledge in } \\
\text { identifying fake } \\
\text { publishers/journals }\end{array}$ & 85.22 & 86.53 & 74.6 & 83.98 & 63.64 & 63.06 & 76.17 \\
\hline $\begin{array}{c}\text { Slow and hidden } \\
\text { feedback }\end{array}$ & 55.27 & 56.58 & 54.69 & 54.03 & 53.6 & 53.02 & 54.53 \\
\hline Lack of Speed & 71.22 & 62.53 & 50.66 & 69.98 & 61.64 & 61.06 & 62.85 \\
\hline Lack of Search skills & 67.19 & 65.5 & 36.61 & 65.95 & 55.61 & 55.03 & 57.65 \\
\hline Lack of Time & 63.09 & 64.45 & 52.51 & 61.85 & 61.51 & 60.93 & 60.72 \\
\hline Lack of Interest & 42.09 & 43.4 & 31.51 & 40.85 & 50.51 & 49.93 & 43.05 \\
\hline
\end{tabular}

The study of the problems of other institutions revealed the fact that the respondents from all the institutions under study faced similar type of problems only with a marginal difference in percentage. However the major problems uniformly identified in the order of priority on the whole include Lack of Authenticity, Lack of knowledge in identifying predatory publishers/journals, Lack of subject coverage, Lack of Quality, Lack of Search skills, Nonavailability of full text, Lack of Time, Lack of Speed, Inactive Links, Lack of up-to-date information, Incomplete bibliographic information, Slow and hidden feedback, Inadequate search facility, Lack of user friendliness, and Lack of Interest. (Table 9)

\section{FINDINGS AND CONCLUSION}

The study throws light on various aspects related to the awareness and use of open access resources for teaching-learning and research purposes. The investigation has brought out a few fruitful facts and figures. It was found that the level of awareness on the open access resources meant for academic related activities is quite encouraging. In addition, the open access resources accessible through online have been utilized significantly. The use of academic social networking sites, open access journals, e-books, online lecture videos, etc was found to be quite encouraging. However, a meager portion of the respondents expressed that they did not use open access resources while another portion of the respondents revealed that they were not even aware of some open access resources. Majority of the respondents have used very little some important sources which are very essential for academic and research purposes such as Plagiarism Detection Tools, Manuscript Editing Tools, and Reference Management Tools. The respondents also had some constraints in using open access resources such as inadequate search facility, Lack of up-to-date information, Slow and hidden feedback, Lack of subject coverage, Non-availability of full text, and Lack of Speed. These facts brought by the present study are worth considering through which the image of the institutions and the research productivity of the professionals can be certainly enhanced. 
Awareness and Use of Open Access Resources among the Students of Selected Engineering Institutions in Telangana State: A Survey

\section{REFERENCES}

[1] Ahmed, S. M. Z. (2017). A survey of students' use of and satisfaction with university subscribed online resources in two specialized universities in a developing country, 5.

[2] Alhabash, S., \& Ma, M. (2017). A Tale of Four Platforms: Motivations and Uses of Facebook, Twitter, Instagram, and Snapchat Among College Students?. Social Media+Society, 3(1), 2056305117469154.

[3] Bosah, G., Okeji, C. C., \& Baro, E. E. (2017). Perceptions, preferences of scholarly publishing in Open Access journals: A survey of academic librarians in Africa. Digital Library Perspectives, 33(4), 378-394. https://doi.org/10.1108/DLP-03-2017-0011

[4] Duy, Joanna \& Vaughan, Liwen (2015). Usage data for electronic resources: A comparison between locally collected and vendor-provided statistics. The Journal of Academic Librarianship, 29 (1), 16-22.

[5] Isibika, I. S., \& Kavishe, G. F. (2018). Utilisation of subscribed electronic resources by library users in Mzumbe university library, Tanzania. Global Knowledge, Memory and Communication, 67(1/2), 109-125. https://doi.org/10.1108/GKMC-09-2017-0075

[6] Mahadevamurthy, M and Adithyakumar, H. (2015). Electronic Information Resources In: Law Libraries: An Overview. Review of Research, 4(4), 1-10.

[7] Malone, Debbie \& Videon, Carol (2015). Assessing undergraduate use of electronic resources: A quantitative analysis of works cited. Research Strategies, 15(3), 151-158.

[8] Sun, J., Sheng, D., Gu, D., Du, J. T., \& Min, C. (2017). Understanding link sharing tools continuance behavior in social media. Online Information Review, 41(1), 119-133. https://doi.org/10.1108/OIR-08-2015-0261. 